\title{
Automation of Gas Analyzer at Converter Shop in Steel Melt Shop
}

\author{
Alla Varun Kumar ${ }^{1}$, S. Aruna ${ }^{2}$ \\ ${ }^{1}$ Student, Department of Electronics and Communication, Andhra University, Visakhapatnam, \\ Andhra Pradesh, India \\ ${ }^{2}$ Assistant Professor, Department of Electronics and Communication, Andhra University, Visakhapatnam, \\ Andhra Pradesh, India
}

\begin{abstract}
The main objective of the project "Automation of Gas Analyzers at Converter shop in Steel Melt Shop" is to analyze the concentration of $\mathrm{CO}, \mathrm{CO}_{2}, \mathrm{H}_{2}$ and $\mathrm{O}_{2}$ present in the converter gas at different locations namely Dry Gas Analyzer, Wet Gas Analyzer and Common Wet Gas Analyzer which is released as a result of BLOWING process. The measurement of $\mathrm{CO}$ concentration in Dry Gas Analyzer is important for the Gas Recovery, storage and distribution of Gas to different customers for heating of Furnaces. The measurement of $\mathrm{CO}_{2}$ concentration in Dry Gas Analyzer is important to control the entry of air into the system and also for reducing the excessive heat generated in the Gas duct. The measurement of $\mathrm{H}_{2}$ concentration in Dry Gas Analyzer is important to know the leakages of water into the Converter to prevent explosion by stopping the blow. The measurement of $\mathrm{O}_{2}$ concentration in Wet Gas Analyzer and Common Wet Gas Analyzer is important to control the entry of air into the system and also to prevent formation of explosive mixture in the system leading to unsafe conditions like a massive explosion, if the percentage of oxygen goes beyond $2 \%$. From the ID Fan the Gas is sent either to the Flare stack for flaring or to the Gas Holder for storing the gas based on CO concentration $\left(\mathrm{CO}>30 \%\right.$ and $\left.\mathrm{O}_{2}<2 \%\right)$. The release gas is sampled before scrubber in hot condition by Dry Gas Analyzer system to measure $\mathrm{CO}, \mathrm{CO}_{2} \& \mathrm{H}_{2}$ concentrations. The gas concentration after ID Fan before flare stack is measured using Wet Gas Analyzer to measure concentration of Oxygen percentage. The Common Wet Gas Analyzer is installed in the common gas duct to Gas recovery plant to measure concentration of Oxygen percentage to prevent air entry into the gas holder and to stop the blowing process and gas recovery in case $\mathrm{O}_{2}$ concentration is above $2 \%$.
\end{abstract}

Keywords: Converter shop, Gas Analyzer

\section{Introduction}

This present project was successfully completed at Steel Melt Shop which is a crucial unit in a Steel Plant. Steel Melt Shop consists of a converter where in the hot metal from the blast furnace is converted to liquid steel by De-carbonation process. Hot metal is actually a mixture of impurities such as Carbon $(3.5-$ $4.25 \%$ ) Silicon (0.4\% - 0.5\%), Manganese (0.3 - 0.4\%), Sulphur (0.04\% max) and phosphorous (0.09\% max). This will be at a temperature of 1300-1400 degrees centigrade. Hot metal is again combined with iron ore, coke, calcium, lime and dolomite and heated to $1700 \mathrm{Deg} \mathrm{C}$ with the aid of combustible gas, oxygen. Oxygen is induced into the converter via Lance which is a pipe housing made of special steel with cooling water circulation to inhibit it from melting at such a high temperature. Inside Lance there exists a housing of spiral tubes through which cooling water is circulated to prevent damage. Induction of oxygen increases the temperature to approximately $1700^{\circ} \mathrm{C}$. Pressure of Oxygen is approximately $16 \mathrm{Kg} / \mathrm{Cm}^{2}$ to $18 \mathrm{Kg} / \mathrm{Cm}^{2}$. This passage of oxygen through Lance is carried on for a duration of 16 to 18 minutes, which is known as "Blowing" Process. During this Blowing process de-carbonation takes place and final yield will be Liquid Steel. During this Blowing process waste gases, which are noxious, are released. They are ought to be cleaned before releasing into atmosphere or storing for reuse. The cleaning of the Gas is done with Gas Cleaning Plant (GCP). Based on the composition of the gas the gas is either recovered and stored in the Gas Holder for further use in the Re-Heating furnaces of Rolling Mills or sent to the atmosphere after burning. Two identical GCPs are installed in SMS for the two converters for collection, cooling, cleaning and recovery of gas and dust.

The gas going through the installation during the Blowing process has a high percentage of $\mathrm{CO}$ which is a combustible, useful gas. During the non-blowing period, air is sucked through the installation. For safety reasons, it is necessary to avoid formation of air gas mixture at the beginning and end of blow, and also during non-blowing period. A natural buffer of inert gas provides safety of operation. The inert buffer is obtained by keeping the Venturi damper wide open during the transient periods at the beginning and end of each blowing sequence. The beginning of the blowing sequence produces a considerable air intake at converter mouth, which causes complete combustion of the refining gas in the hood. Following this phase the actuation of the draft control system gradually closes the scrubber and reduces the flow of induced air at the hood, until operating 
characteristics without combustion are obtained. Thus, during this period, there is a phase corresponding to the stoichiometric combustion of the gas during which all the oxygen of the induced air is used to burn the entire $\mathrm{CO}$ emitted by the bath. The resultant gas is then only composed with Carbon Dioxide $\left(\mathrm{CO}_{2}\right)$ and Nitrogen $\left(\mathrm{N}_{2}\right)$, and is therefore completely inert with respect to the air removed from the installation and also with respect to the gas collected without combustion. The same phenomenon occurs, in the reverse direction, at the end of the blowing sequence. As a result, in all cases an intermediate inert gas buffer whose volume is sufficient to fully purge the installation separates the air and the combustible gas.

In the above process it becomes mandatory for the measurement of concentration of $\mathrm{CO}, \mathrm{CO}_{2}, \mathrm{H}_{2}$ and $\mathrm{O}_{2}$ for safe operation of the plant. Calorific Value of produced Gas from Converter Blowing Process is about $2000 \mathrm{KCAL} / \mathrm{NM}^{3}$

The composition of Gas being generated from Converter blowing process is:

$\begin{array}{ll}\text { Carbon monoxide } & 60 \text { to } 80 \% \\ \text { Hydrogen } & 2.0 \text { to } 3.0 \% \\ \text { Carbon dioxide } & 10 \text { to } 20.0 \% \\ \text { Remaining: } & \mathrm{N}_{2}, \mathrm{O}_{2} \text { and other gases }\end{array}$

II. Description Of Gas Analyzers

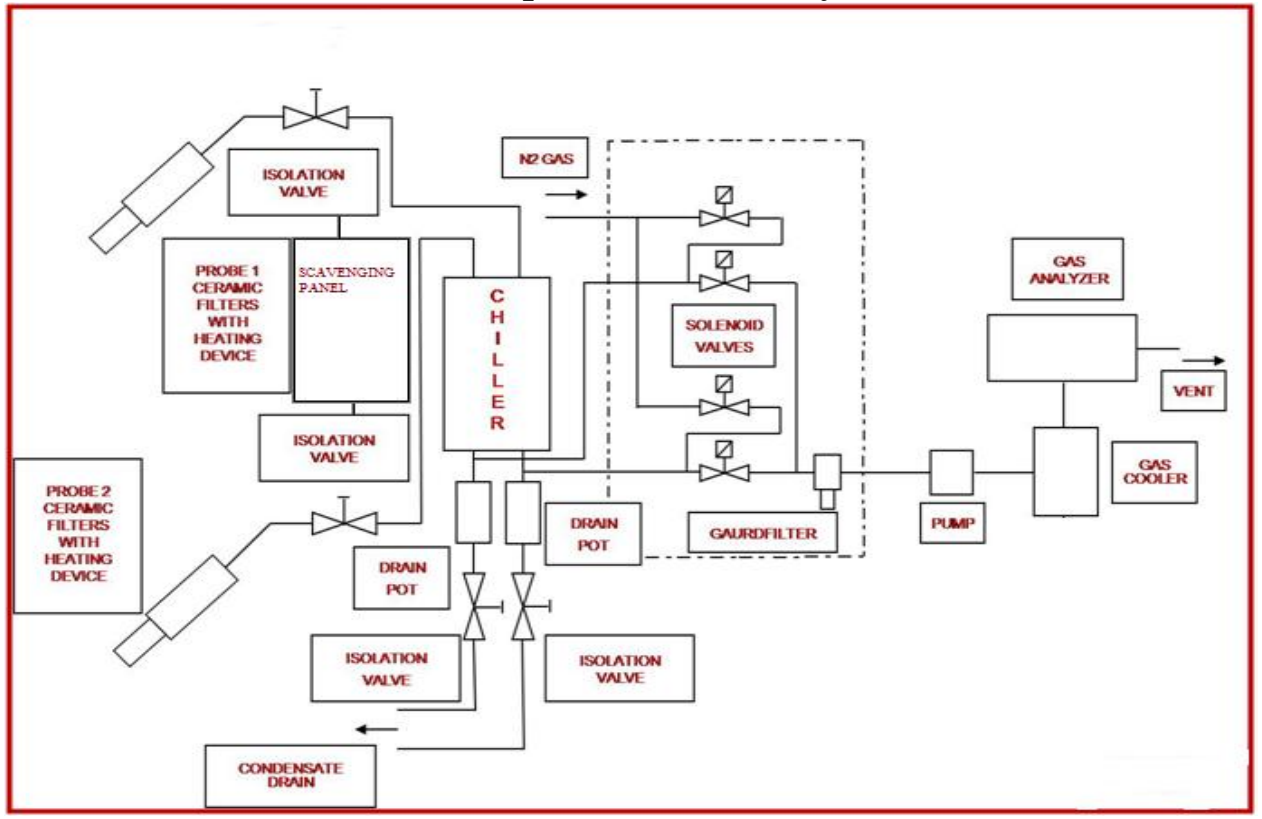

Fig 1. Block Diagram repersenation of Gas Analyzers

\subsection{Gas Analysis System Parts}

The Gas Analyzer System consists of the following parts

1. The HS Probe Assembly - 2 Nos.

2. The Scavenging Panel - 1 No.

3. The Analysis Panel - 1 No.

\subsubsection{The Special Probe Assembly}

The probe assembly is used for collecting from main gas duct for sending to gas analyzers for analysis of gas.

Each Probe Assembly consists of:

1. HS Probe Bushing Tube

2. HS Probe Ceramic Filter

3. HS Probe Head

4. HS Probe Tube Assembly

5. Ring Heater

\subsubsection{The Scavenging Panel}

The scavenging panel is a collection of solenoid valves for both the probes. It consists of solenoid valves for both the probes. It consists of solenoid valves for purging and sampling. The main function of scavenging panel is to collect the gas from the probe head and send it to analyzer panel for analysis. The other function of scavenging panel is to purge both the sampling lines and probes through solenoid valves to ensure 
cleanliness of probes, filters and pipe lines. The scavenging panel is installed near the probe. The maximum recommended distance from the probe to scavenging panel is 5 meters. A purging gas ( Nitrogen) free from moisture through is connected to the panel. The nitrogen @ 6bar is connected to PG01; the 1/2" Line from probe to the scavenging panel is connected to OP01 and OP02. A 1/2" line from Probe is to connected to IP01 and IP02.A 10 core X 105 square $\mathrm{mm}^{2} \mathrm{Cu}$, flexible armored from analysis Panel to scavenging panel needs to be drawn for the operation of the solenoid valves. A 2 core X $1.5 \mathrm{~mm}^{2}$ cable needs to be drawn for the connection of the pressure switch. It is to be noted that the difference between the scavenging panel and the probe is to be kept minimum as possible so that there is less pressure drop and the cleaning is effective.

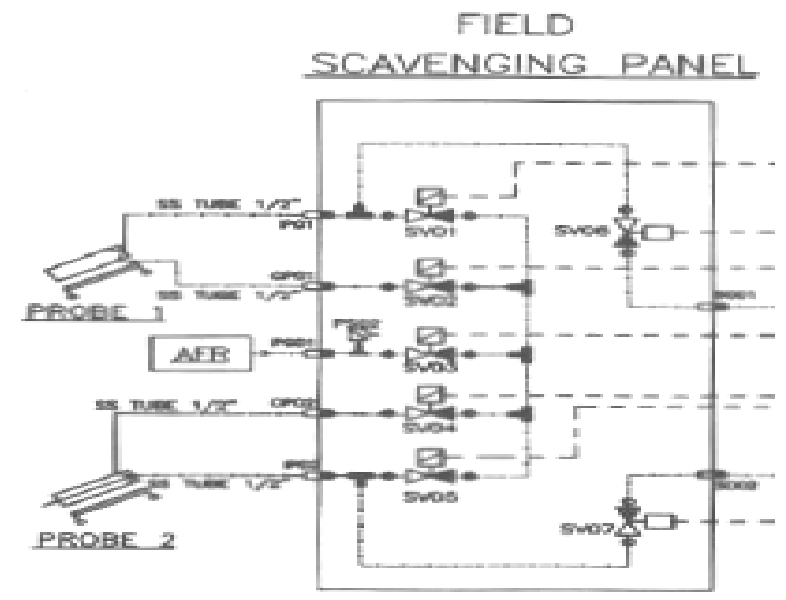

Fig 2. Scavenging Panel

\subsubsection{Analysis Panel}

The analyzer panel is used to collect gas from the scavenging panel, cool the gas and send the gas to different analyzers $\left(\mathrm{CO}, \mathrm{CO}_{2}, \mathrm{H}_{2}\right.$ and $\left.\mathrm{O}_{2}\right)$ for analysis.

The analyzer panel consists of the following

1. Solenoid valves for calibration and sampling

2. Diaphragm pumps for collection of sample gas from gas duct

3. Gas coolers for condensation of gas moisture in the sample gas.

4. Peristaltic pumps for draining of condensed water in the gas coolers.

5. Vent pumps for venting of gas to the atmosphere and keep the other probe ready for sampling.

6. Rota meters for supplying required flow (60lit/hr) to individual gas analyzers

7. Condensate monitors with filter for sensing the moisture content in sampling gas. A condensate high alarm will stop the gas analysis forcing the blow stoppage.

8. PLC system consists of CPU, power supply, digital input, digital output and analog input modules. This PLC does the following functions

- It houses the logic required for both manual and automatic operation of the sampling system.

- It takes all the field inputs and processes it

- It gives outputs for operation of solenoids, pumps, etc.

- It displays information of all inputs and outputs through panel display unit.

- Through panel HMI interface clocking of system is possible in manual mode.

9. Gas analyzers- $\mathrm{CO}, \mathrm{CO}_{2}, \mathrm{H}_{2}$ and $\mathrm{O}_{2}$ for analyzing concentrations of different gases.

10. Calibration set up with solenoids and gas cylinders for calibration of different gas analyzers $\left(\mathrm{CO}, \mathrm{CO}_{2}, \mathrm{H}_{2}\right.$ and $\mathrm{O}_{2}$ ).

\subsection{Functioing Of Oxygen Analyzer- Oxymat}

In contrast to almost all the other gases, oxygen is paramagnetic. This property is utilized as the measuring principle by the OXYMAT channel.Oxygen molecules in an in homogeneous magnetic field are drawn in the direction of the increased field strength due to their paramagnetism. When two gases with different oxygen concentrations meet in a magnetic field, a pressure difference is produced between them. 


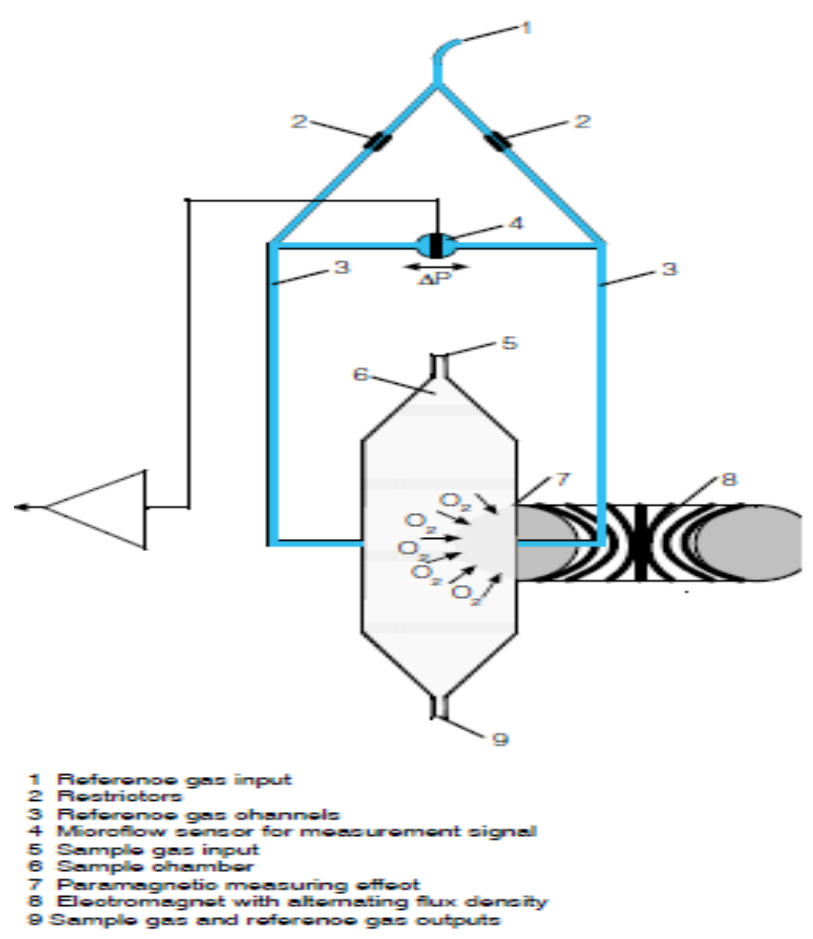

Fig 3. Oxymat Mode of Operation

For the Oxymat channel, one of the gases is a reference gas $\left(\mathrm{N}_{2}, \mathrm{O}_{2}\right.$ or air), the other is the sample gas. The reference gas is introduced into the sample chamber through two channels. One of these reference gas streams meets the sample gas within the area of a magnetic field. Because the two channels are connected, the pressure, which is proportional to the oxygen concentration, causes a flow. This flow is converted into an electric signal by a micro flow sensor. The micro flow sensor consists of two nickel grids heated to approx. $120^{\circ} \mathrm{C}$ which form a Wheatstone bridge together with two supplementary resistors. The pulsating flow results in a change in the resistance of the $\mathrm{Ni}$ grids. This results in a bridge offset which depends on the oxygen concentration in the sample gas. Because the flow sensor is located in the reference gas stream, the measurement is not influenced by the thermal conductivity, the specific heat or the thermal friction of the sample gas. This also provides a high degree of corrosion resistance because the flow sensor is not exposed to the direct influence of the sample gas.

By using a magnetic field with alternating strength, the effect of the background flow in the micro flow sensor is not detected, and the measurement is thus independent of the instrument orientation. The sample chamber is directly in the sample path and has a small volume. There is therefore a very short response time for the OXYMAT. After the analysis of oxygen the sample gas is vented out through the sample gas outlet

\subsection{Description And Functioning Of $\mathrm{Co}$ And $\mathrm{Co}_{2}$ Analyzer- Ultramat}

An IR source (7) at $600^{\circ} \mathrm{C}$ emits an infrared radiation which is modulated with $81 / 3 \mathrm{~Hz}$ by a chopper(5). After passing the sample cell(4), the intensity of the radiation is measured by the detector $(11,12)$. The represented detector is composed of layers filled with the component to be analyzed. The energy of the middles of the IR bands of the measured gases is mainly absorbed in the first layer. The second layer absorbs the edge energy which is tuned on high selectively in the third layer via an aperture. 


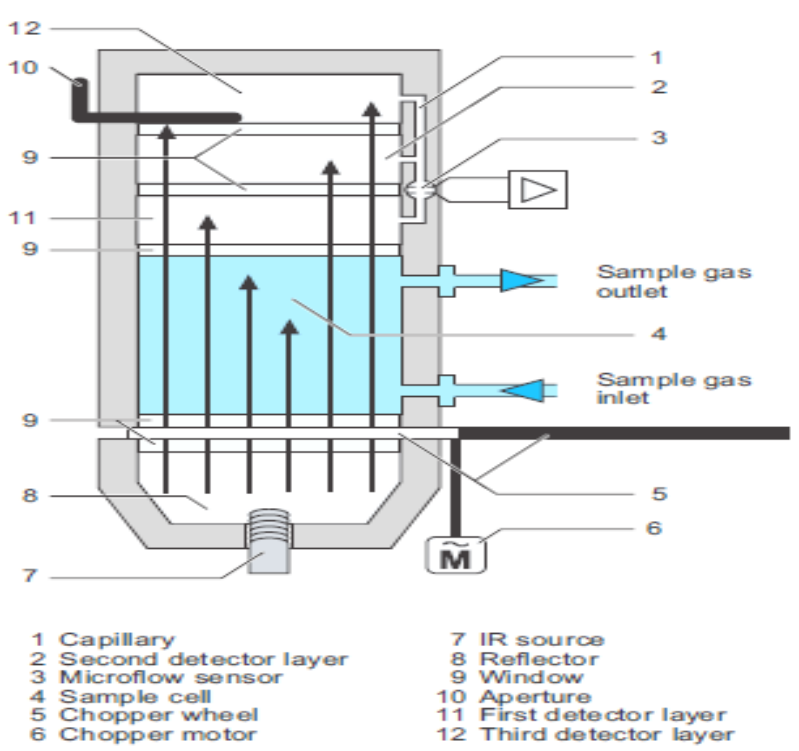

Fig 4. Ultramat Mode of Operation

When passing through the layers the radiation absorption results in different pressure increases and so to a flow via the capillary hole. The micro flow sensor there generates a signal which is nearly independent of the interferences from the components at the band edges.This analyzer uses ambient air for auto calibration. Only once in a year or in 18 months calibration is required with a certified cylinder.

\subsection{Description And Functioning Of Hydrogen Analyzer- Calomat}

The measuring principle is based on the different thermal conductivity of gases. The CALOMAT operates with a micro- mechanically manufactured Si chip whose measuring diaphragm contains thin-film resistors.The resistors are regulated at a constant temperature. A current is required to achieve this, and its magnitude assumes a particular value depending on the thermal conductivity of a sample gas. This raw value is subjected to further electronic processing and is used to calculate the gas concentration. To suppress ambient temperature influences, the sensor is located in a thermostatically- controlled stainless steel housing. To prevent flow influences, the sensor is only subjected to an indirect flow.

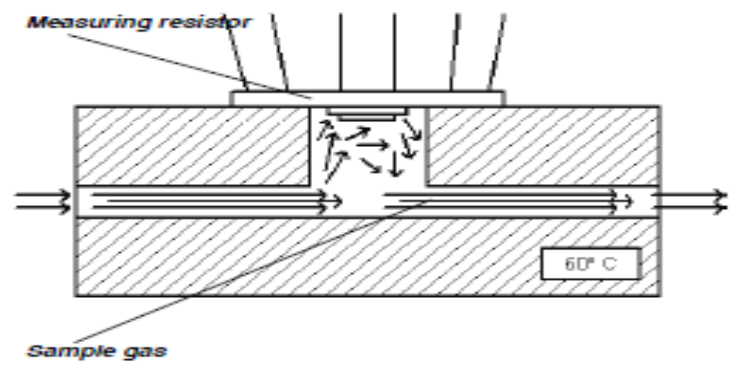

Fig 5. Calomat Mode of Operation

\section{Existing And New Methods Of Controlling Of Sampling System}

3.1 Existing System Description

In the old system of Gas analyzers the sampling system is controlled through manual selector switches. All the operations are to be done from the local panel only. Except for the \% of Gas compositions no other inputs available to the operator doing process control from Main control room located at far off place from the Gas Analyzer room. Because of this Maintenance persons have to run to the Gas Analyzer rooms located at very far off areas from control room to attend to any fault in the system. This is taking plenty of time leading to long production delays. Also safety of Converter shop is entirely dependent on reliable functioning of Gas Analyzers frequent problems are causing great difficulty in blowing affecting production.

\section{Disadvantages:}

$>$ Frequent failure of manual switches

$>$ No connectivity of faults to main PLC system in Main control room. 


\section{$>$ Frequent breakdowns}

$>$ Frequent Production delays.

To overcome these difficulties it is decided to replace the manual sampling system with PLV based system for Automatic operation and communication with Main PLC of Converter Shop.

\subsection{New System Description}

The system is designed to operate in Auto mode on power up. On power up the system goes to measuring mode. The system stays in measuring mode. The system stays in the measuring mode as per the cycle time selected in the touch screen on the front door. Probe 1 goes to the standby measuring mode and probe 2 to the cleaning mode. But the active measuring will start when Lance in contact will come in operation. After the period already given by operator the probe changing takes place. The active probe goes for cleaning. The other one goes for standby measuring mode and waits for lance in contact.

\subsubsection{Measuring Mode}

In the measuring mode if lance in contact comes, the gas is sucked from the tapping point through the HS probe assembly mounted on the process through the sample gas pump in the Analyzer panel. The gas is first filtered by the ceramic filter, which filters the sample of all the dust. The sample gas is heated by the probe with the help of the ring heater which heats the ceramic filter, keeping it dry to effective cleaning. The sample gas now travels through the sample line to the Scavenging panel. In the scavenging panel SV06 is opened during measuring. The gas travels through SV06 and comes out through the bulk head union SO01. The gas is then transported through the sample line to the analyzer panel. In the analyzer panel the heated sample gas which has high dew points enters the cooler. The cooler is maintained at a temperature of $4-8^{\circ} \mathrm{C}$. The sample gas with high dew point, is cooled suddenly as it enters the cooler and looses all its moisture in the cooler.

The moisture drained out of the cooler is collected in the condensate vessel and a auto condensate drain pump which operates intermittently ( i.e it is switched On for $2 \mathrm{sec}$ and switched OFF for $8 \mathrm{~min}$, cyclically by the PLC) removes the condensate from the condensate vessel. The timing of the auto condensate drain pump can be changed through the two line display according to the moisture load. The sample gas which now comes out of the cooler, is dry without any moisture. The gas now passes through the solenoid valves to the condensate monitor, which filters dust upto 1 micron and gives a signal to the PLC via a switching unit for any presence of moisture the gas coming out of the condensate monitor is dust and moisture free gas.

During the sampling time of Probe 1, Probe 2 remains in the bypass mode, once the timing of probe 1 is over probe 2 comes into the sampling and probe 1 after cleaning sequence goes to the bypass mode. The probes being in the bypass mode eliminates the transportation delay caused by the changeover of the probes and the analyzer can immediately start giving actual on the changeover. These all happen whenever lance in contact comes into the operation.

The sample gas now goes to the analyzer through the flow meter. The flow meters are set at 72-80 LPH. During operation if there is an occurrence of low flow due to probe chokage. Or chokage in the cooler, the system automatically goes to the cleaning mode. The flow sensor in the flow meter detects the low flow. If the cause of flow is eliminated after cleaning the system runs normally but if the fault is persistent the system stops and gives an alarm contact to the CCR.

\subsubsection{Cleaning Mode}

Probe 1 Cleaning: When probe 1 is in cleaning mode SV06 is closed, SV01 opens to let the Nitrogen in and SV03 operates in pulses to complete the inner purging. After the inner purging SV02 opens and SV03 again operates in pulses to compete the outer purging.

Probe 2 Cleaning: When probe 2 is in the cleaning mode SV07 is closed, SV05 opens to let the nitrogen in and SV03 operates in pulses to complete the inner purging. After the inner purging SV04 opens and SV03 operates in pulses to complete the outer purging.

\subsection{Dry Gas Analyzer}

\section{Functional Description Of Gas Analyzers}

Dry Gas Analyzer is installed at below 54 meter level in Converter shop. This is used to measure concentration of $\mathrm{CO}, \mathrm{CO}_{2}, \mathrm{H}_{2}$ and $\mathrm{O}_{2}$ in gas generated in converter blowing process. As the gas is in hot condition at a temperature of approximately $800-1000^{\circ} \mathrm{C}$ it is called Dry gas and hence the system is called Dry Gas analyzer system. The system basically measures concentration of $\mathrm{CO}_{2}$ when blowing process starts. The blowing is done using oxygenate at flow rate of about $500 \mathrm{NM}^{3} / \mathrm{min}$ using water cooled lance. As the blow starts, due to decarbonization process blowing oxygen reacts with carbon in the liquid metal and forms $\mathrm{CO}$ gas. A part of this $\mathrm{CO}$ gas is converted into $\mathrm{CO}_{2}$ due to burning of part of released $\mathrm{CO}$ gas with air in the process initially for a period of $2 \mathrm{~min}$. When $\mathrm{CO}_{2}$ crosses more than $15 \%$, the skirt above the converter is moved close to 
the converter mouth. This stops entering of air into the system and burning of CO gas is reduced. This results in reduction of carbon dioxide gas from $25 \%$ to $10 \%$. In this phase $\mathrm{CO}$ starts picking and reaches above $30 \%$ in about 2 min. During the start of blow due to air ingress $\mathrm{O}_{2}$ also will increase to $20 \%$ and will come down lo less than $2 \%$ once $\mathrm{CO}$ starts picking up above $30 \%$. During normal blow $\mathrm{O}_{2}$ concentration will be usually at around $0.2 \%$. During blow due to any air ingress or leakage in the system the $\mathrm{O}_{2}$ will increase and if it is above $2 \%$ anytime after $2 \mathrm{~min}$ of blow start the blowing process will be stopped as it may cause explosive mixture along with formed $\mathrm{CO}$ gas.

During blow the dry gas analyzer system also measures concentration of $\mathrm{H}_{2} \%$ in the gas produced in the converter. The cooling gas duct which carries the released gas from converter comprises stack of cooling pipes attached together and water is circulated through them. Any damage or burst of tubes will result in water entering into the converter. As converter holds the liquid metal, the entry of water into it will cause formation of steam and it may try to escape causing huge explosion. To prevent this $\mathrm{H}_{2}$ analyzer is used to measure the concentration of hydrogen which is indirect measure of water leakage into converter. At any given point of time if $\mathrm{H}_{2}$ concentration is above $7 \%$ in the measuring gas blow will be stopped in converter. Another source of high $\mathrm{H}_{2}$ is leakage of water from converter blowing lance which carries water for cooling the lance while blowing with $\mathrm{O}_{2}$. Thus $\mathrm{H}_{2}$ analyzer plays major role in keeping the safety of the converter shop.

The concentration of $\mathrm{CO}$ gas in released gas from blowing process determine whether it can be reused or can be flared to atmosphere. If the CO concentration of released gas is above $30 \%$ then the gas can be utilized for heating the furnaces in other plants. Hence if $\mathrm{CO} \%$ is above $30 \%$ it will be sent to gas holder through switch over station and will be distributed to further mills for heating of furnaces etc. if CO\% is below $30 \%$ it will be sent to flare stack for passing into atmosphere through switch over station and will be flared out. This is active only during blowing process.

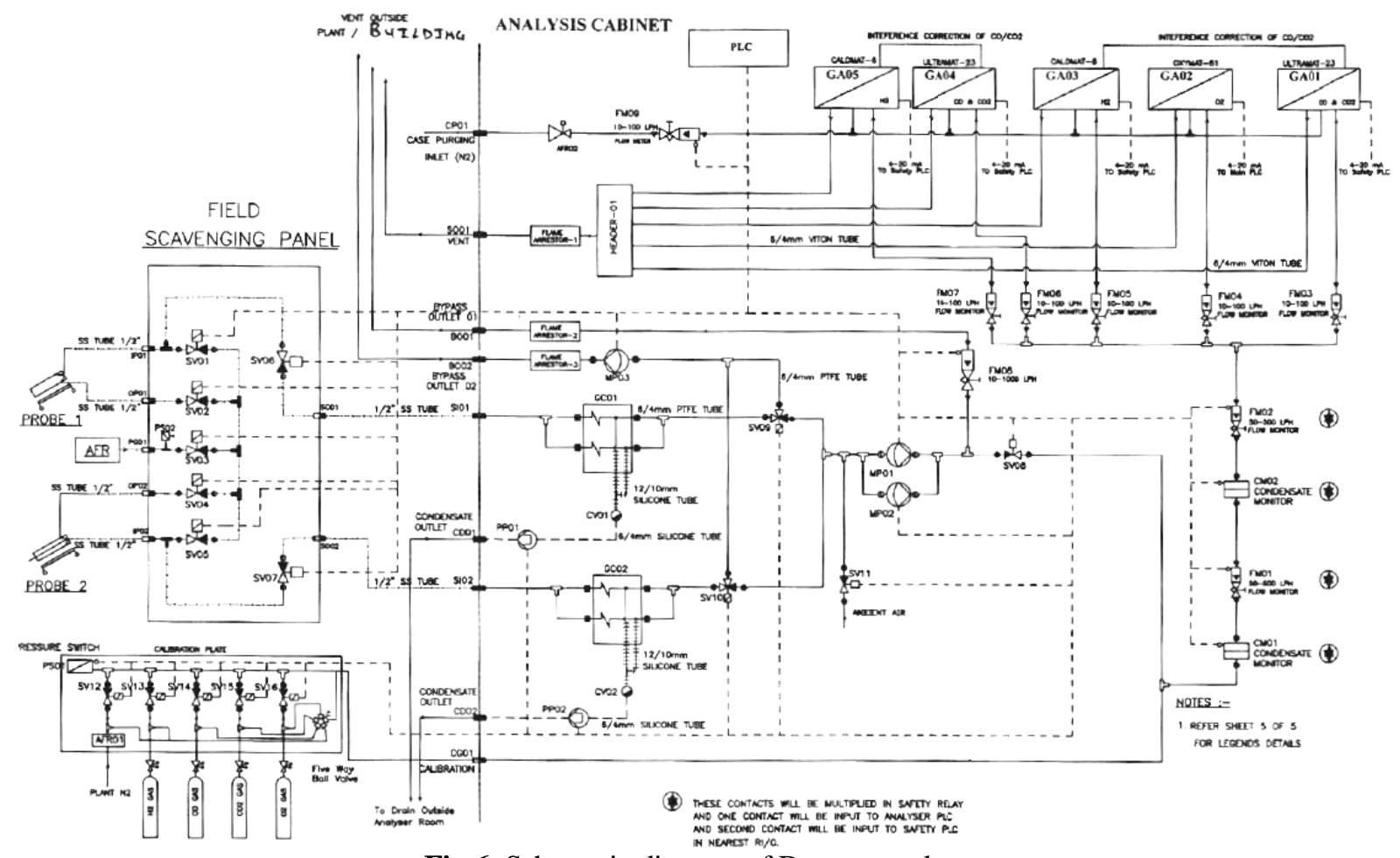

Fig 6. Schematic diagram of Dry gas analyzer

\subsection{Wet Gas Analyzer}

Wet Gas analyzer is installed at the gas duct between ID fan and the flare stack. This system is used to measure the concentration of $\mathrm{O}_{2}$ which is very critical for ascertaining leakages (air entry) into the system from cooling stack upto ID fan inlet pipe carrying converter gas. Any leakage of air into the system after dry gas analyzer makes $\mathrm{O}_{2}$ high in the gas carrying $\mathrm{CO}$ gas. This becomes explosive mixture and may cause explosion in the gas duct if any spark is generated. Thus measurement of $\mathrm{O}_{2}$ concentration is very critical for knowing the healthiness of gas duct between dry gas analyzer and ID fan. If $\mathrm{O}_{2}$ concentration is above $2 \%$ anytime after $2 \mathrm{~min}$ of blow start then blowing process is stopped. In addition to $\mathrm{O}_{2}, \mathrm{CO} / \mathrm{CO}_{2}$ concentration is also measured in wet gas analyzer. This is active only during blowing process. 
The Wet Gas Analyzer is placed at Omtr level on the ground after the ID fan. During this Blowing process de-carbonation takes place and final yield will be Liquid Steel, along with it waste gases, which are noxious are also released which traverses through skirt, hood, stack and cleaned / cooled at scrubber and further moves through venturi for flow measurement and finally reaches the ID Fan where the wet gas is analyzed for the oxygen concentration using redundant probes for sampling gas, field scavenging panel to route gas through sampling lines to the wet gas analyzer system.

The sample gas enters into the unique gas analyzer Ultramat having Auto Cal with Ambient Air, which is designed to detect the percentage of $\mathrm{CO}, \mathrm{CO}_{2}$ in the sample gas. The sample gas also enters parallely into Oxymat analyzer to detect the percentage of $\mathrm{O}_{2}$ in the sample gas.

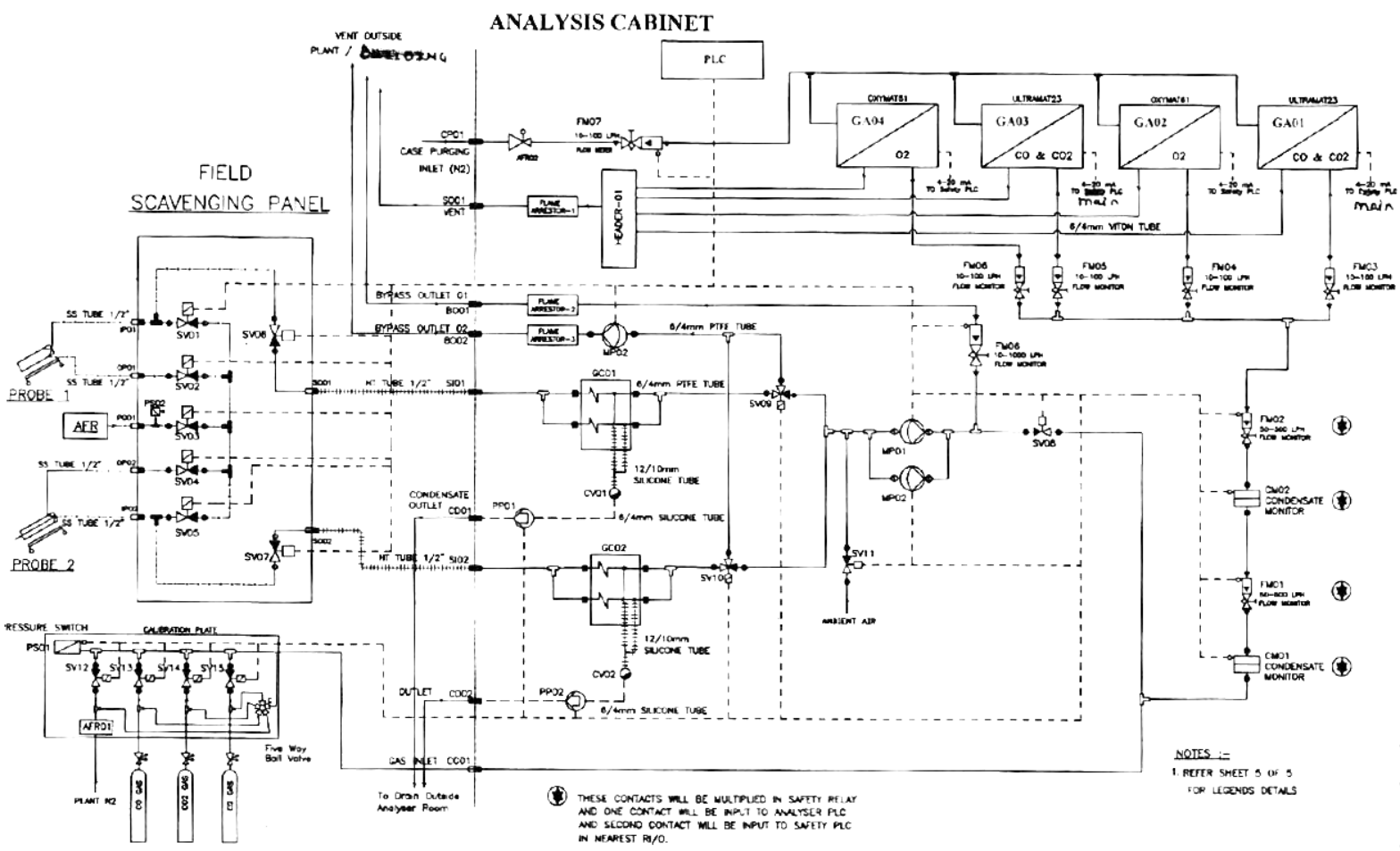

Fig 7. Schematic diagram of Wet gas analyzer

\subsection{Common Wet Gas Analyzer}

The gas duct of all the converters are joined to a single pipeline for carrying gas to Gas Recovery Plant. In the common gas duct to the gas holder, the Common Wet Gas Analyzer is installed and it is used to measure $\mathrm{O}_{2}$ concentration in the common gas duct. This analysis is active throughout the year irrespective of blowing process. This measures $\mathrm{O}_{2}$ in common line and whether blowing is in progress at any converter, this will run continuously and stops blowing in all the converters if $\mathrm{O}_{2}$ is above $2 \%$. This will also stop sending gas to gas recovery plant and closes the water seal check valve of both the converters in case of high $\mathrm{O}_{2}$. This will also close the inlet valve of Gas Holder.

The Common Wet Gas Analyzer is present in the common duct where the gases from different converters are mixed into an common line which is sent to gas recovery. The sample gas enters the gas analyzer Oxymat analyzer to detect the percentage of $\mathrm{O}_{2}$ in the sample gas.

Also the LD gas is rich in carbon monoxide which is a potential fuel. So this gas is recovered for usage in furnaces, etc. So if the recovered gas contains oxygen beyond $2 \%$, an explosive mixture is formed. Hence we also stop the recovery of the gas (Immediate Stop Recovery) and blowing process in case common wet gas analyzer oxygen is above $2 \%$

If gas recovery is not stopped then gas with high concentration of oxygen is stored in gas holder along with converter gas. This mixture is prone to explosion if there is any external spark. If an explosion will take place, then there will be huge loss to the plant. 


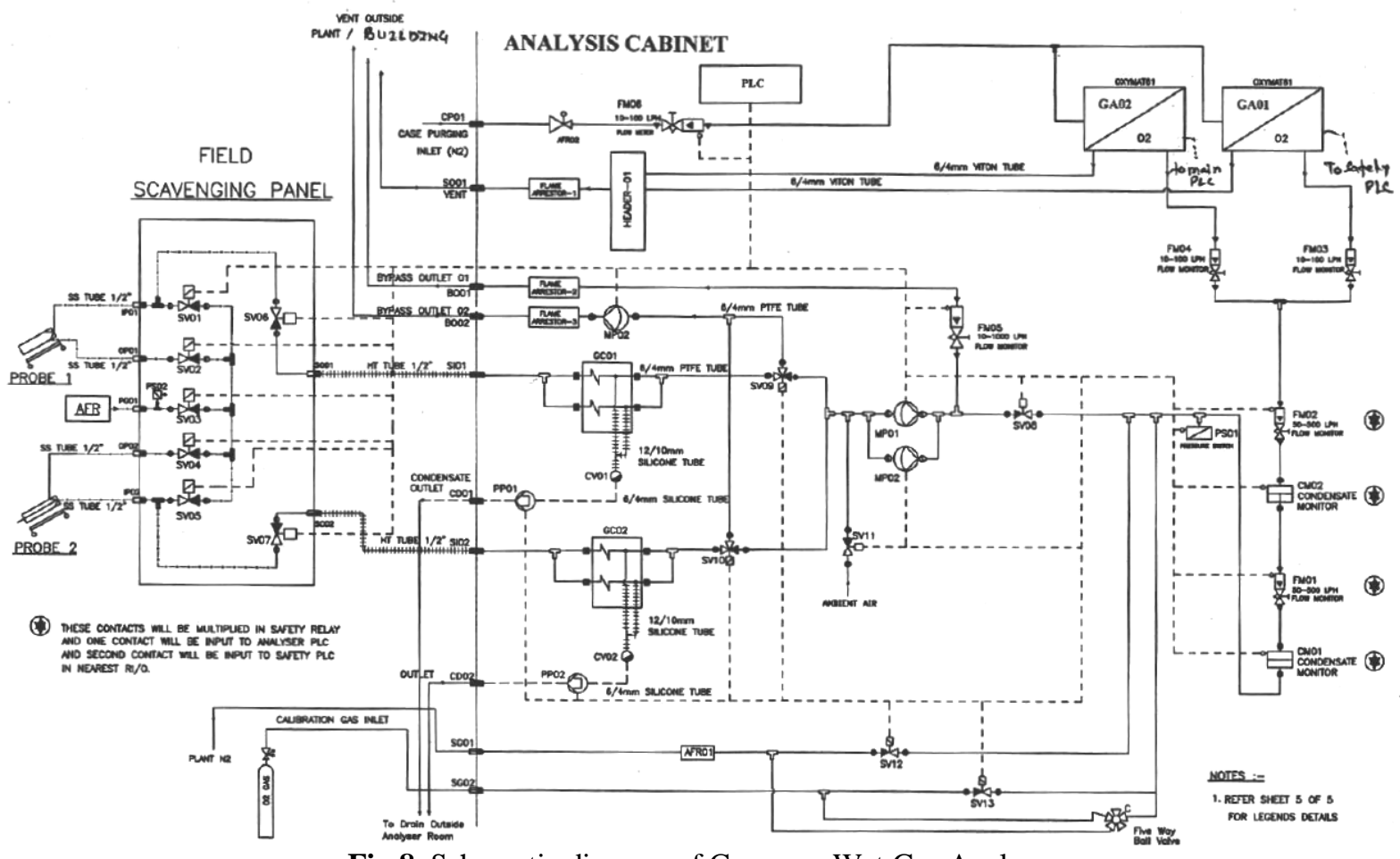

Fig 8. Schematic diagram of Common Wet Gas Analyzer

\section{Programmable Logic Controller}

\subsection{Basic Requirments}

In order to create or change a program, the following items are needed:

$\checkmark$ PLC

$\checkmark$ Programming Device

$\checkmark$ Programming Software

$\checkmark$ Connector Cable

\subsubsection{Programming Devices}

The program is created in a programming device (PG) and then transferred to the PLC. The program for the S7-200 can be created using a dedicated Siemens SIMATIC S7 programming device, such as a PG 720 or PG 740. A personal computer (PC), with STEP 7 Micro/WIN installed, can also be used as a programming device with the S7-200

\subsubsection{Software}

A software program is required in order to tell the PLC what instructions it must follow. Programming software is typically PLC specific. A software package for one PLC, or one family of PLCs, such as the S7 family, would not be useful on other PLCs. The S7-200 uses a Windows based software program called STEP 7-Micro/WIN32. The PG 720 and PG 740 have STEP 7 software pre-installed. Micro/WIN32 is installed on a personal computer in a similar manner to any other computer software.

\subsubsection{Connector Cables PPI}

Connector cables are required to transfer data from the (Point-to-Point Interface) programming device to the PLC. Communication can only take place when the two devices speak the same language or protocol. Communication between a Siemens programming device and the S7-200 is referred to as PPI protocol (point topoint interface). An appropriate cable is required for a programming device such as a PG 720 or PG 740. The S7-200 uses a 9-pin, D-connector. This is a straight-through serial device that is compatible with Siemens programming devices (MPI port) and is a standard connector for other serial interfaces.

A special cable, referred to as a PC/PPI cable, is needed when a personal computer is used as a programming device. This cable allows the serial interface of the PLC to communicate with the RS-232 serial interface of a personal computer. DIP switches on the PC/PPI cable are used to select an appropriate speed (baud rate) at which information is passed between the PLC and the computer. 


\subsection{S7-200 Executes Its Tasks in a Scan Cycle}

The S7-200 executes a series of tasks repetitively. This cyclical execution of tasks is called the scan cycle.

$\checkmark$ Reading the inputs: The S7-200 copies the state of the physical inputs to the process-image input register.

$\checkmark$ Executing the control logic in the program: The S7-200 executes the instructions of the program and stores the values in the various memory areas.

$\checkmark$ Processing any communications requests: The S7-200 performs any tasks required for communications.

$\checkmark$ Executing the CPU self-test diagnostics: The S7-200 ensures that the firmware, the program memory, and any expansion modules are working properly.

$\checkmark$ Writing to the outputs: The values stored in the process-image output register are written to the physical outputs.

\subsection{Operating Mode Of S7-200 CPU}

The S7-200 has two modes of operation: STOP mode and RUN mode. The status LEDs on the front of the CPU indicates the current mode of operation. In STOP mode, the S7-200 is not executing the program, and program and CPU configuration can be downloaded. In RUN mode, the S7-200 is running the program.

$\checkmark$ The S7-200 provides a mode switch for changing the mode of operation. The mode switch is used to manually select the operating mode. Setting the mode switch to STOP mode stops the execution of the program; setting the mode switch to RUN mode starts the execution of the program; and setting the mode switch to TERM (terminal) mode does not change the operating mode.

If a power cycle occurs when the mode switch is set to either STOP or TERM, the S7-200 goes automatically to STOP mode when power is restored. If a power cycle occurs when the mode switch is set to RUN, the S7-200 goes to RUN mode when power is restored.

$\checkmark$ STEP 7--Micro/WIN allows you to change the operating mode of the online S7-200. To enable the software to change the operating mode, manually the mode switch is set on the S7-200 to either TERM or RUN. You can use the PLC > STOP or PLC > RUN menu commands or the associated buttons on the toolbar to change the operating mode.

$\checkmark$ STOP instruction is inserted in the program to change the S7-200 to STOP mode. This is used to halt the execution of your program based on the program logic.

\subsection{Step 7 Micro/Win}

1. The simple, easy to learn programming software under Windows 200/XP for the SIMATIC S7-200

2. A large number of wizards support he programming even of difficult automation task.

3. For fast startup and timesaving programming

4. With large scope of functions

5. Based on standard Windows software (user interface similar to standard applications, such as Microsoft Word, Outlook)

6. With 3 standard editors STL, LAD and CSF; you can switch between these editors at any time

7. Generation, exporting and importing of user-specific libraries (including standard commands and userdefined subroutines)

8. Documentation CD with manuals, software tools and example programs as support

\subsection{Dry Gas Analyzer Trend}

\section{Results}

The trends of the Dry Gas Analyzer are plotted with Time on the X-axis and the percentage or volume of gas on the $\mathrm{Y}$-axis depending on the selection. The time scaling duration on the $\mathrm{X}$-axis is $30 \mathrm{~min}$ and the percentage scaling on the $\mathrm{Y}$-axis varies with different gases. The scaling for different gases is mentioned below 1. $\mathrm{H}_{2}$ gas- $0 \%$ to $10 \%$

2. CO gas- $0 \%$ to $100 \%$

3. $\mathrm{CO}_{2}$ gas- $0 \%$ to $40 \%$

4. $\mathrm{O}_{2}$ gas- $0 \%$ to $25 \%$

5. Lance $\mathrm{O}_{2}$ flow- 0 to $650 \mathrm{Nm}^{3} / \mathrm{hr}$ 


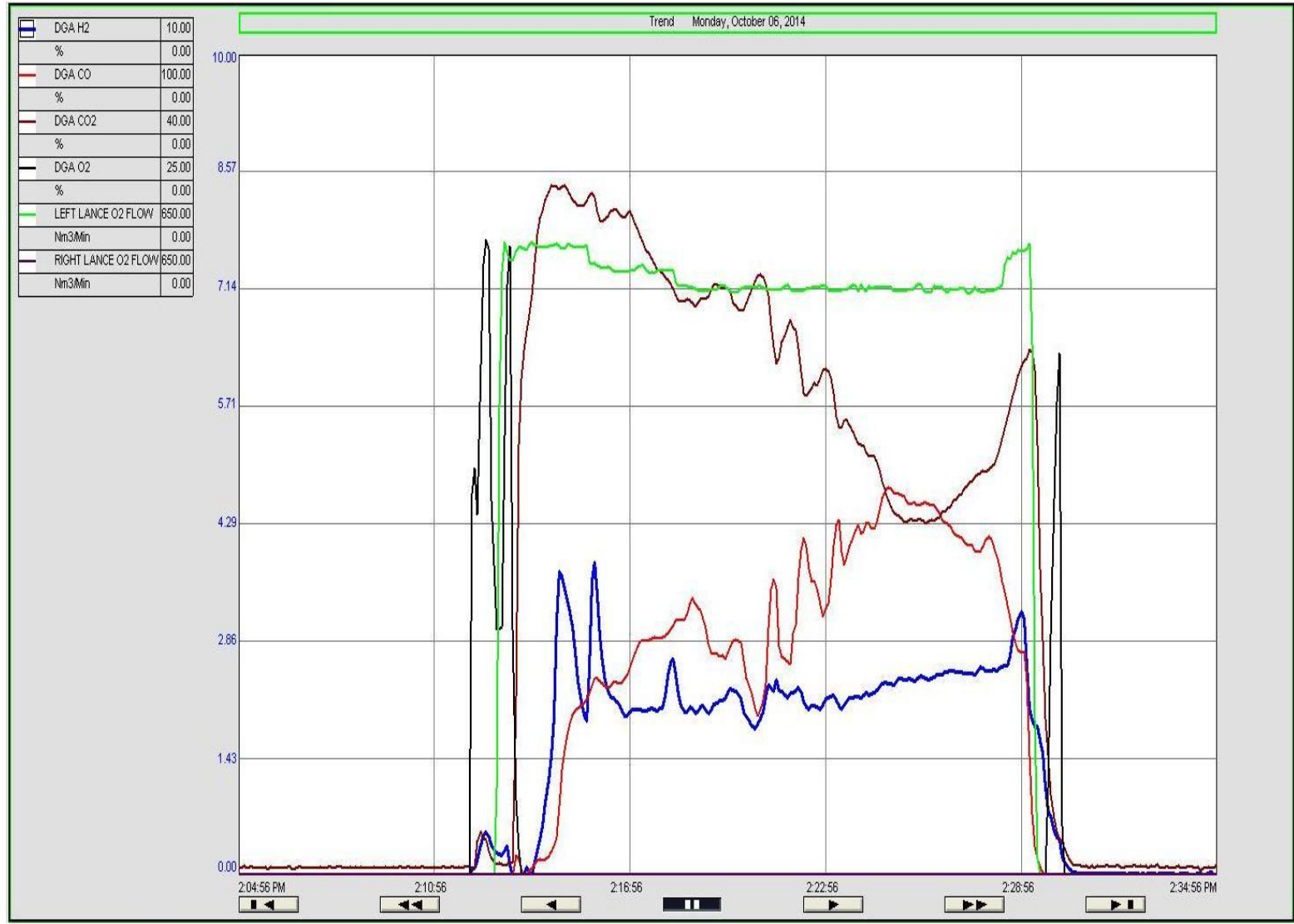

Fig 9. Dry Gas Analyzer Trend

\subsection{Wet Gas And Common Wet Gas Analyzer Trends}

The trends of the Wet Gas Analyzer are plotted between Time on the X-axis and percentage or volume of gas on the $\mathrm{Y}$-axis depending on the selection. The time duration the $\mathrm{X}$-axis is $30 \mathrm{~min}$ and on the $\mathrm{Y}$-axis the scaling varies for different gases. The scaling of the gas flow are mentioned below

1. Wet gas analyzer $\mathrm{O}_{2}$ gas- $0 \%$ to $25 \%$

2. Lance $\mathrm{O}_{2}$ flow- 0 to $650 \mathrm{Nm}^{3} / \mathrm{hr}$

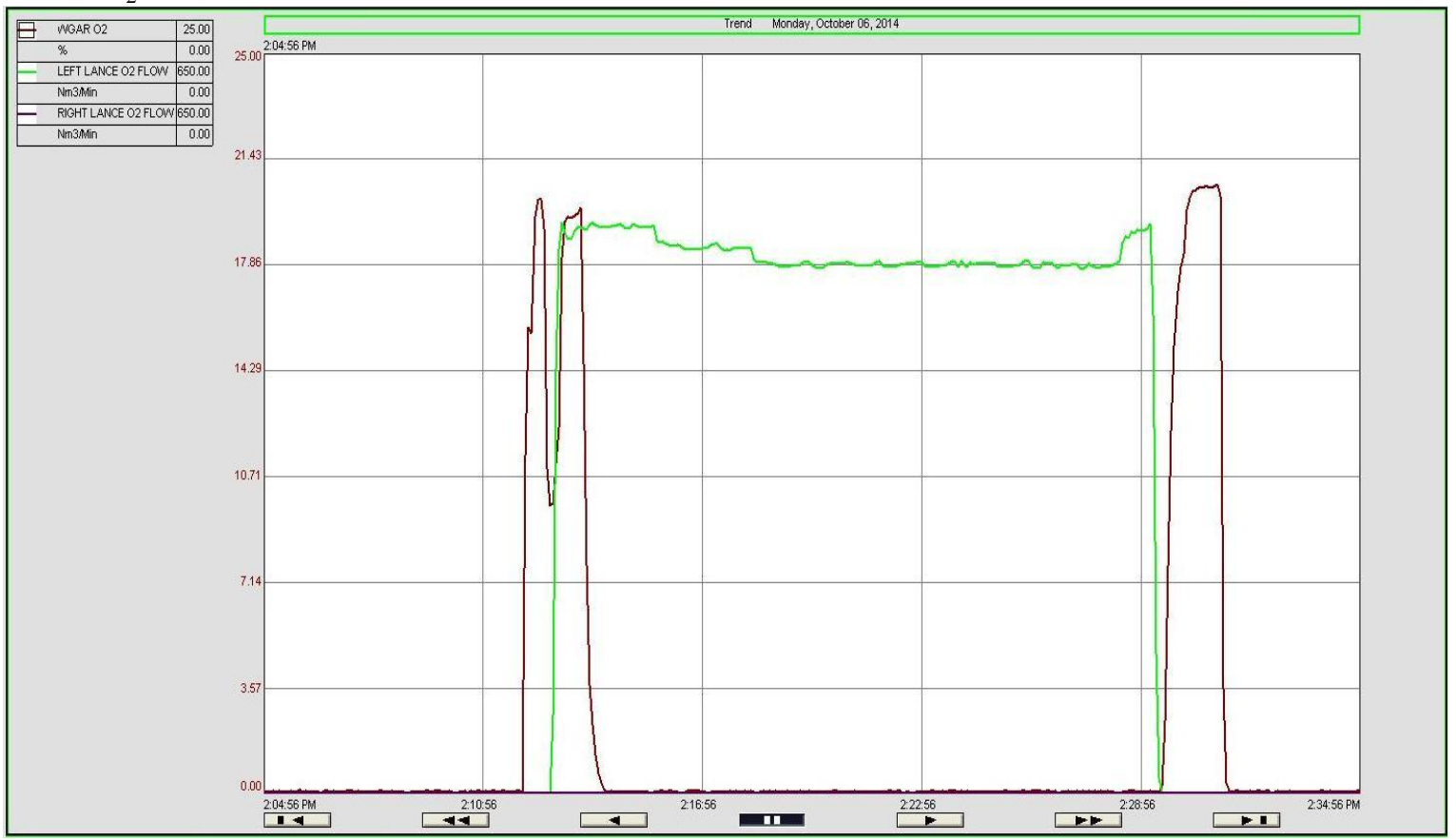

Fig 10. Wet Gas Analyzer Trend 


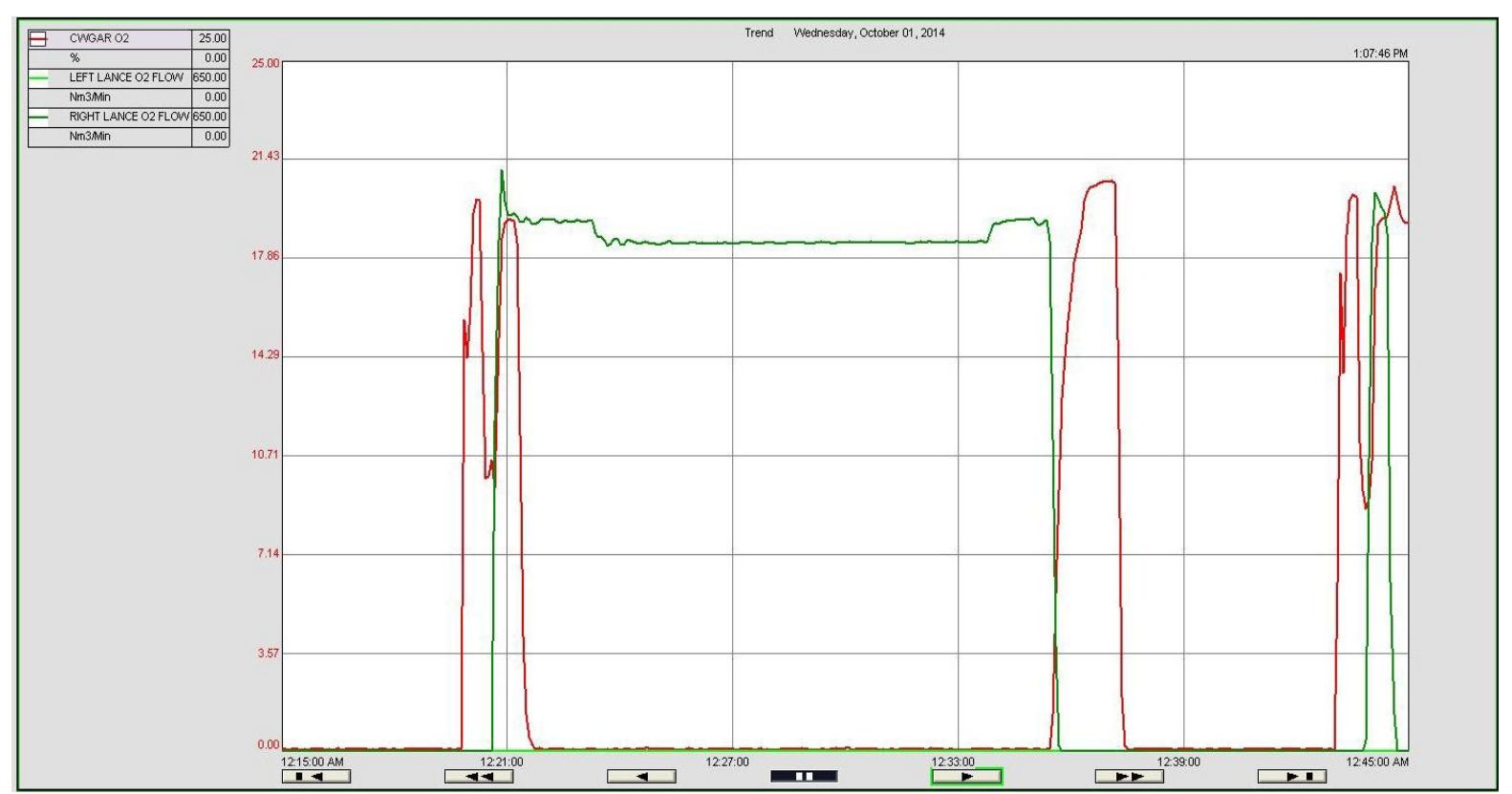

Fig 11. Common Wet Gas Analyzer Trend

VII. Conclusion

When the blowing is started the Main Converter PLC gives contact to start the analysis. With the development of logic in PLC the Manual operation is totally eliminated. Due to flexibility of ladder logic any modification in logic is easier to implement any changes in future. It is very easy to develop the program in new PLC as all the registers, counters, timers, etc., are available with a mouse click. The new logic helped in eliminating manual intervention and operation. This helped in obtaining faster result compared to the old Manual System. This also helped in easy identification of the problems and faster trouble shooting. This has improved the performance of the gas analysis system and ensured continuous availability of the system. This is helped in maintaining good calorific value of gas and quality of steel as reliability of the system improved and frequent failures are eliminated.

With the implementation of Automatic logic in DRY GAS ANALYZER the performance of sampling system has improved and safety to process and personnel is ensured with continuous availability of $\mathrm{CO}, \mathrm{CO} 2$ and $\mathrm{H}_{2}$ analysis.

With the implementation of Automatic logic in WET GAS ANALYZER the performance of sampling system has improved and safety to process and equipment is ensured with continuous availability of $\mathrm{O} 2$ analysis.

With the implementation of Automatic logic in Common WET GAS ANALYZER the performance of sampling system has improved and safety to process and Gas recovery plant is ensured with continuous availability of $\mathrm{O} 2$ analysis in the common duct.

\section{References}

[1]. Programmable Logic Controllers by Krishna Kant

[2]. Programmable Logic Controller (PLC) Tutorial, Siemens Schematic S7-200 by Stephen P. Tubbs

[3]. HANDBOOK OF ANALYTICAL INSTRUMENTS, SECOND EDITION By DR R.S. KHANDPUR

[4]. Automating Manufacturing Systems with PLC's, version 4.7,@ 2009; Hugh Jack

[5]. SCADA Systems by Robert Lemos.

[6]. Oxygen Gas Analyzer OXYMAT Manual.

[7]. Hydrogen Gas Analyzer CALOMAT Manual.

[8]. Carbon Gas Analyzer ULTRAMAT Manual.

[9]. SMS Converter Operating Manual.

[10]. Converter GCP Operating Manual. 